\title{
基于萘酰亚胺的次氯酸荧光探针的合成及细胞成像性能研究
}

\author{
王欣宇 ${ }^{\dagger}, a, c$ \\ 戚少龙 ${ }^{\dagger, a, c}$ \\ 杜建时*,a,c \\ 李强 $b, c$ \\ 祝录宝 $b, c$ \\ 薛龙启 $b, c$ \\ 赵 晴 $a, d$ \\ 杨清虎 $*, b, c$ \\ 李耀先 ${ }^{b}$ \\ 丛宪玲 ${ }^{a}$ \\ ( ${ }^{a}$ 吉林大学中日联谊医院 长春 130033) \\ ( ${ }^{b}$ 吉林大学化学学院 长春 130061) \\ ( ${ }^{c}$ 吉林大学中日联谊医院 吉林省淋巴外科重点实验室 吉林省淋巴外科工程实验室 长春 130033) \\ $\left({ }^{d}\right.$ 吉林大学中日联谊医院 吉林省记忆与认知障碍疾病工程实验室 长春 130031)
}

\begin{abstract}
摘要 以荎酰亚胺结构为荧光发色团, 设计开发了一种含 $\mathrm{C}=\mathrm{C}$ 双键的、具有分子内电荷转移(ICT)效应的新型水溶性 优化的次氯酸荧光探针 3-(2-㲵基丙烯酸乙酯基)-4-羟基- $N$-正丙基-1,8-菜酰亚胺(NAEC). 添加次氯酸后, 探针分子 $\mathrm{NAEC}$ 中的 $\mathrm{C}=\mathrm{C}$ 双键被氧化, 生成醛基, 探针 NAEC 原有的 ICT 效应被破坏, 产生荧光信号. 经核磁、质谱、苂光发 射光谱和 UV-Vis 吸收光谱对其结构和检测性能进行了研究. 结果表明, 在 $\mathrm{pH}=7.4$ 的 $N, N-$ 二甲基甲酰胺(DMF)/磷酸缓 冲盐溶液 $(\mathrm{PBS})(V: V=1: 19)$ 缓冲体系中, 探针 NAEC 可在 $10 \mathrm{~s}$ 内完成对次氯酸的检测, 苂光分析检测限为 2.4 $\mathrm{nmol} / \mathrm{L}$, 斯托克斯位移为 $100 \mathrm{~nm}$; 探针 NAEC 显示出较强的抗干扰性, 能在其他活性氧、小分子生物硫醇及常见阴离 子等 22 种干扰物存在下完成次氯酸的专一检测. 同时, 该探针分子的膜透性与生物相容性良好, 具备较好的活体内源 性 $\mathrm{ClO}^{-}$荧光成像能力, 在生物检测及环境监控等领域具有良好的应用前景.
\end{abstract}

关键词 萗酰亚胺; 次氯酸; 苂光探针; 细胞成像

\section{A Naphthalimide-Based Hypochlorous Acid-Selective Fluorescent Probe and Its Application in Cell Imaging}

\author{
Wang, Xinyu ${ }^{\dagger, a, c}$ \\ Qi, Shaolong ${ }^{\dagger, a, c}$ \\ Du, Jianshi ${ }^{*, a, c}$ \\ Li, Qiang ${ }^{b, c}$ \\ Zhu, Lubao ${ }^{b, c}$ \\ Xue, Longqi ${ }^{b, c}$ \\ Zhao, Qing ${ }^{a, d}$ \\ Yang, Qingbiao*,b,c \\ Li, Yaoxian ${ }^{b}$ \\ Cong, Xianling ${ }^{a}$ \\ $\left({ }^{a}\right.$ China-Japan Union Hospital of Jilin University, Changchun 130061) \\ ( ${ }^{b}$ College of Chemistry, Jilin University, Changchun 130061) \\ ( ${ }^{c}$ Key Laboratory of Lymphatic Surgery Jilin Province, Engineering Laboratory of Lymphatic Surgery Jilin Province, Chi- \\ na-Japan Union Hospital of Jilin University, Changchun 130031) \\ $\left({ }^{d}\right.$ Engineering Laboratory of Memory and Cognitive Impairment Disease Jilin Province, China-Japan Union Hospital of Jilin \\ University, Changchun 130031)
}

\begin{abstract}
A novel naphthalimide-based fluorescence probe ethyl (E)-3-(2-butyl-6-hydroxy-1,3-dioxo-2,3-dihydro-1H-benzo[de]isoquinolin-5-yl)-2-cyanoacrylate (NAEC) containing $\mathrm{C}=\mathrm{C}$ double bond group with intramolecular charge transfer (ICT) effect for hypochloric acid detection was synthesized, which could selectively and sensitively respond to hypochloric acid within $10 \mathrm{~s}$ in phosphate buffer saline (PBS) buffer solution $[N, N$-dimethylformamide (DMF)/PBS $(V: V=1: 19), \mathrm{pH}=$ 7.4]. The mechanism is that the $\mathrm{C}=\mathrm{C}$ double bond in the probe can be oxidized and turned to aldehyde group, then the original ICT effect of probe is destroyed, and the fluorescence of naphthalimide restores. The spectroscopic properties of the probe NAEC towards $\mathrm{ClO}^{-}$were studied through UV-vis absorption and fluorescence emission spectroscopies. The results showed that the probe NAEC possessed a large pseudo Stokes shift $(100 \mathrm{~nm})$ and the limit of detection (LOD) for NAEC has been estimated to be $2.4 \mathrm{nmol} / \mathrm{L}$. In addition, the probe exhibited good response and anti-interference performance for hypochloric
\end{abstract}

* Corresponding authors. E-mail: dujs@jlu.edu.cn; yangqb@jlu.edu.cn

Received August 6, 2020; revised September 1, 2020; published online September 22, 2020.

Project supported by the Key Laboratory Construction Project of Jilin Province (No. 20190901002JC), the Natural Science Foundation of Jilin Province (No. 20200201169JC) and the Innovation Building Projects of Jilin Province (No. 2019C007).

吉林省重点实验室建设项目(No. 20190901002JC)、吉林省自然科学基金(No. 20200201169JC)和吉林省创新能力建设(No. 2019C007)资助项目.

共同第一作者(These authors contributed equally to this work). 
acid in the presence of 22 kinds of interferences, such as other reactive oxygen, small molecule biosulphol and common anions. And probe NAEC exhibited good cell permeability and was successfully applied in living cells by fluorescence imaging. The method is expected to be extended to the diagnosis of diseases and the detection in environmental samples, etc.

Keywords naphthalimide; hypochlorous acid; fluorescence probe; cellular imaging

次氯酸 $(\mathrm{HClO}) /$ 次氯酸盐 $\left(\mathrm{ClO}^{-}\right)$因其强氧化性被作 为漂白剂和消毒剂在生活中得以广泛应用，如饮用水的 消毒和医院等公共场所的常规杀菌等 ${ }^{[1]}$. 此外, 次氯酸 ( $\mathrm{HClO})$ 也是一种生物体内重要的活性氧物质(ROS), 在 生物体免疫过程中发挥着不可或缺的作用, 当细菌入侵 人体或人体表面皮肤受损时, 单核细胞、巨噬细胞和嗜 中性粒细胞等白细胞内的过氧化物和氯化物在髓过氧 化物酶(MPO)的作用下生成 $\mathrm{HClO}$, 利用其强氧化性可 清除多种病原微生物 ${ }^{[2-3]}$. 然而, 过度的 $\mathrm{ClO}^{-}$可能导致 组织损伤和各种人类疾病, 如关节炎、心血管疾病、肾 脏疾病、神经退化和癌症等 ${ }^{[4-6]}$. 因此, $\mathrm{ClO}^{-}$的浓度可以 作为衡量生命体健康状况的重要指标. 但 $\mathrm{ClO}^{-}$的胞内 浓度较低, 且反应活性高 ${ }^{[3]}$, 使得目前检测生物体内 $\mathrm{ClO}^{-}$浓度和分布状况较为困难, 所以开发一种可靠且 有效的监测生命体系中的 $\mathrm{ClO}^{-}$的方法具有重要意义.

近年来, 对于 $\mathrm{ClO}^{-}$的定性和定量分析, 已经开发了 许多科学方法, 包括质谱法 ${ }^{[7]}$ 、高效液相色谱法(HPLC) 分析 ${ }^{[8]}$ 和生物分析方法 ${ }^{[9-10]}$ 等. 其中, 苂光探针法因其 简便快捷、选择性高、灵敏度高和实时监控等优点, 被 认为是非侵入和就地识别 $\mathrm{ClO}^{-}$的最强大、最有效的工 具 ${ }^{[11-13]}$. 最近, Peng 课题组 ${ }^{[14]}$ 基于氟嗍二吡咯甲川 (BODIPY)合成了一种检测癌细胞中 $\mathrm{ClO}^{-}$的超灵敏荧光 探针, Duan 课题组 ${ }^{[15]}$ 开发出具有高亮度和良好光稳定 性的 $\mathrm{ClO}^{-}$近红外苂光探针, 李春霞课题组 ${ }^{[16]}$ 合成了基 于亚甲基蓝的探针分子用于细胞内源性 $\mathrm{ClO}^{-}$的检测. 上述探针虽检测效果较好, 但存在如下问题: (1)多数探 针分子的斯托克斯位移较小, 荧光信号可能受到自吸收 和内滤效应的干扰; (2)多数探针的水溶性不佳, 需要在 大比例有机溶剂体系中发挥作用, 很难用于细胞和生物 检测; (3)部分探针只能在 $\mathrm{ClO}^{-}$浓度范围比较窄的区间 内进行定量测定, 并且响应时间相对较长等.

䒺酰亚胺具有较大的 Stocks 位移和较理想的氧化 还原性能, 并且光学和化学性能稳定, 易于修饰, 是设 计荧光探针的常用荧光发色团 ${ }^{[2]}$, 但目前用于次氯酸检 测的相关工作较少, 本工作利用次氯酸对特定结构 $\mathrm{C}=$ $\mathrm{C}$ 双键的氧化特性, 从菜酰亚胺和氧基乙酸乙酯出发, 设计开发了一种新型苂光探针分子 3-(2-氰基丙烯酸乙 酯基)-4-羟基- $N$-正丙基-1,8-䒬酰亚胺(NAEC), 该探针 具有快速响应、斯托克斯位移较大、水溶性好、生物低 毒性等优点, 可实现细胞中 $\mathrm{ClO}^{-}$的实时苂光定量检测. 合成路线如 Scheme 1 所示.

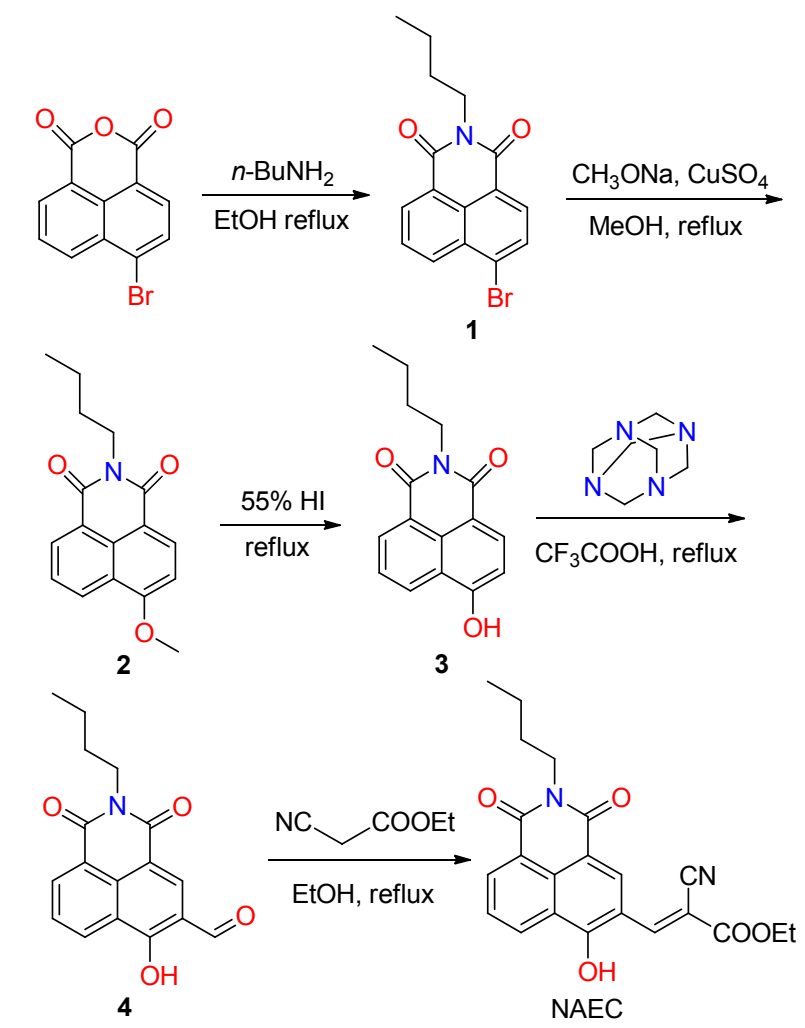

图式 1 探针 NAEC 合成路线

Scheme 1 Synthetic route of the probe NAEC

\section{1 结果与讨论}

\section{$1.1 \mathrm{pH}$ 值对探针 NAEC 检测性能的影响及响应时间}

为了探究溶液酸碱性对探针 NAEC 检测效果的影 响, 首先考察了探针分子苂光强度在不同溶液 $\mathrm{pH}$ 值下 的变化情况. 如图 1A 所示, 在 $\mathrm{pH}$ 值分别为 $4 \sim 10$ 的一 组 PBS 缓冲溶液中, 探针的苂光强度无明显变化, 说明 探针分子具有较好的环境酸碱耐受性; 加入过量 $\mathrm{ClO}^{-}$ 后，溶液荧光强度随 $\mathrm{pH}$ 值的升高而逐渐增大, $\mathrm{pH}=7.4$ 左右达到最大. 之后随着 $\mathrm{pH}$ 值的进一步增大, 苂光强 度逐渐下降. 考虑到生物体内环境普遍为中性和弱碱 性，因此选定测试溶液 $\mathrm{pH}$ 值为 7.4.

为了考察探针对检测底物的响应速度, 在探针溶液 中加入 $\mathrm{ClO}^{-}$后测定了苂光强度的变化情况(图 1B), 结 果发现, 苂光强度在加入 $\mathrm{ClO}^{-}$后 $10 \mathrm{~s}$ 内即达到最大值. 说明, 探针 NAEC 的检测速度较快, 能够实时监测 $\mathrm{ClO}^{-}$ 的浓度变化.

\section{2 探针 NAEC 对 $\mathrm{ClO}^{-}$的紫外-可见光谱响应}

图 2 为探针 $\mathrm{NAEC}$ 紫外-可见吸收光谱随 $\mathrm{ClO}^{-}$浓度 

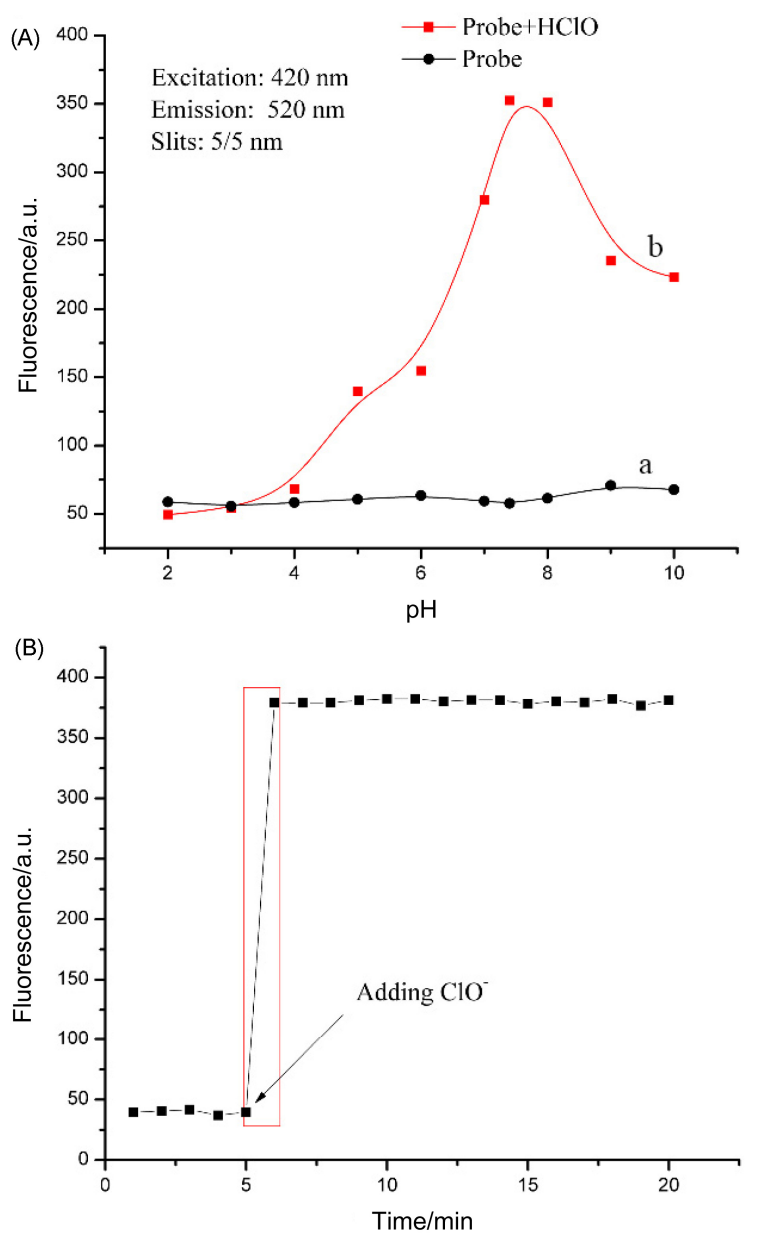

图 1 (A)探针 NAEC $\left(5.0 \times 10^{-6} \mathrm{~mol} / \mathrm{L}\right)$ 溶液在加入 $\mathrm{ClO}^{-}$前 (a)、后(b)苂光光谱随溶液 $\mathrm{pH}$ 值的变化情况[DMF/PBS $(V$ : $V=1: 19), \lambda_{\mathrm{ex}}=420 \mathrm{~nm}, \lambda_{\mathrm{em}}=520 \mathrm{~nm}$, slit: $5 / 5 \mathrm{~nm}$ ]和(B)加入 $\mathrm{ClO}^{-}$后荧光强度随时间变化情况 $[\mathrm{DMF} / \mathrm{PBS}(V: V=1: 19)$, $\mathrm{pH}=7.4, \lambda_{\mathrm{ex}}=420 \mathrm{~nm}, \lambda_{\mathrm{em}}=520 \mathrm{~nm}$, slit: $\left.5 / 5 \mathrm{~nm}\right]$

Figure 1 (A) Fluorescence spectra of NAEC $\left(5.0 \times 10^{-6} \mathrm{~mol} / \mathrm{L}\right)$ before (a) and after (b) addition of $\mathrm{ClO}^{-}$in different $\mathrm{pH}$ conditions [DMF/PBS $(V: V=1: 19), \lambda_{\mathrm{ex}}=420 \mathrm{~nm}, \lambda_{\mathrm{em}}=520 \mathrm{~nm}$, slit: $5 / 5 \mathrm{~nm}]$ and (B) time-dependent fluorescence intensity changes of NAEC $\left(5.0 \times 10^{-6} \mathrm{~mol} / \mathrm{L}\right)$ after addition of $\mathrm{ClO}^{-}$ [DMF/PBS $(V: V=1: 19), \mathrm{pH}=7.4, \lambda_{\mathrm{ex}}=420 \mathrm{~nm}, \lambda_{\mathrm{em}}=520$ nm, slit: $5 / 5 \mathrm{~nm}]$

的变化情况. 结果表明, 在向溶液中加入 $\mathrm{ClO}^{-}$前, NAEC 溶液无明显颜色, 可见区域几乎没有 UV-Vis 吸 收; 加入 $\mathrm{ClO}^{-}$后, NAEC 溶液迅速由无转变黄色, 并在 $450 \mathrm{~nm}$ 附近出现明显吸收峰, 吸收峰随着 $\mathrm{ClO}^{-}$浓度的 增加而增强; 当 $\mathrm{ClO}^{-}$的加入量达到 9.0 equiv. 时, 吸收 峰强度不再发生变化, 达到饱和。

\section{3 探针 NAEC 对 $\mathrm{ClO}^{-}$的荧光光谱响应}

图 3 为探针 NAEC 苂光发射光谱随 $\mathrm{ClO}^{-}$浓度的情 况情况. 结果表明, 在未加入 $\mathrm{ClO}^{-}$时, NAEC 溶液自身 几乎没有苂光发射. 加入 $\mathrm{ClO}^{-}$后, NAEC 溶液在 $520 \mathrm{~nm}$ 附近出现苂光发射峰, 且苂光强度随 $\mathrm{ClO}^{-}$浓度的增加

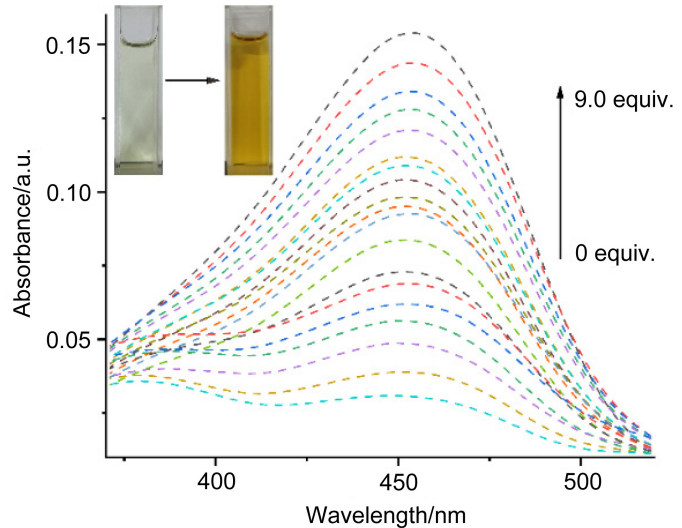

图 2 探针 NAEC $\left(5.0 \times 10^{-6} \mathrm{~mol} / \mathrm{L}\right)$ 在添加不同浓度的 $\mathrm{ClO}^{-}$ $(0 \sim 9$ equiv.) 前后的 UV-Vis 吸收光谱[DMF/PBS $(V: V=1$ : 19), $\mathrm{pH}=7.4$ ]

Figure 2 UV-Vis absorption spectra of probe NAEC $\left(5.0 \times 10^{-6}\right.$ $\mathrm{mol} / \mathrm{L})$ upon addition of different concentrations of $\mathrm{ClO}^{-}(0 \sim 9$ equiv.) [DMF/PBS $(V: V=1: 19), \mathrm{pH}=7.4]$

Insert: emission color changes of probe NAEC $\left(5.0 \times 10^{-6} \mathrm{~mol} / \mathrm{L}\right)$ before and after addition of $\mathrm{ClO}^{-}$(9 equiv.) under a $365 \mathrm{~nm}$ light
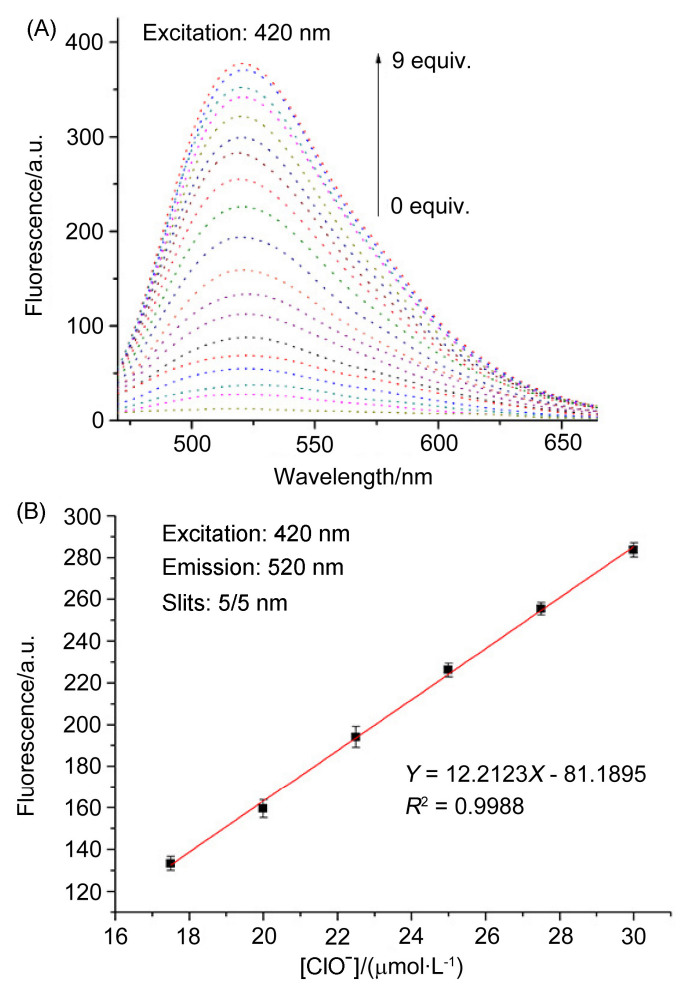

图 3 (A)探针 NAEC 溶液 $\left(5.0 \times 10^{-6} \mathrm{~mol} / \mathrm{L}\right)$ 在添加不同浓度的 $\mathrm{ClO}^{-}(0 \sim 9$ equiv.) 后的苂光光谱[DMF/PBS $(V: V=1: 19)$, $\mathrm{pH}=7.4, \lambda_{\mathrm{ex}}=420 \mathrm{~nm}$, slit: $\left.5 / 5 \mathrm{~nm}\right]$ 和(B)探针 NAEC 的苂光强 度与 $\mathrm{ClO}^{-}$浓度的线性拟合曲线 $[\mathrm{DMF} / \mathrm{PBS}(V: V=1: 19)$, $\mathrm{pH}=7.4, \lambda_{\mathrm{ex}}=420 \mathrm{~nm}, \lambda_{\mathrm{em}}=520 \mathrm{~nm}$, slit: $\left.5 / 5 \mathrm{~nm}\right]$

Figure 3 (A) Fluorescence spectra of probe NAEC $\left(5.0 \times 10^{-6}\right.$ $\mathrm{mol} / \mathrm{L})$ upon addition of different concentrations of $\mathrm{ClO}^{-}(0 \sim 9$ equiv.) $\left[\mathrm{DMF} / \mathrm{PBS}(V: V=1: 19), \mathrm{pH}=7.4, \lambda_{\mathrm{ex}}=420 \mathrm{~nm}\right.$, slit: $5 / 5 \mathrm{~nm}]$ and (B) Linear fit curve of fluorescence intensity of NAEC vs $\mathrm{ClO}^{-}$concentration [DMF/PBS $(V: V=1: 19), \mathrm{pH}=$ $7.4, \lambda_{\mathrm{ex}}=420 \mathrm{~nm}, \lambda_{\mathrm{em}}=520 \mathrm{~nm}$, slit: $\left.5 / 5 \mathrm{~nm}\right]$ 
而逐渐增强，当 $\mathrm{ClO}^{-}$浓度上升至 9.0 equiv. 时，荧光发 射强度达到最大. 以 $\mathrm{ClO}^{-}$浓度为横坐标, NAEC 溶液在 $520 \mathrm{~nm}$ 处苂光强度为纵坐标构图(图 3B), 结果发现, 二 者呈现较好的线性关系 $\left(R^{2}=0.9981\right)$. 线性回归方程: $Y=12.2157 X+81.6816$, 使用 Eq. 1 计算得出探针对 $\mathrm{ClO}^{-}$的荧光检测限 $\mathrm{DL}$ 为 $2.4 \times 10^{-9} \mathrm{~mol} / \mathrm{L}(2.4 \mathrm{nmol} / \mathrm{L})$. 可见探针 NAEC 可对 $\mathrm{ClO}^{-}$进行高灵敏度的定量检测.

$$
\mathrm{DL}=3 \sigma / \kappa
$$

$\sigma$ 是空白溶液的标准偏差, $\kappa$ 是校准曲线的斜率.

\section{4 探针对 $\mathrm{ClO}^{-}$的特异性与干扰性实验}

为了考察 NAEC 对 $\mathrm{ClO}^{-}$的检测专一性及抗干扰能 力, 分别向探针溶液中加入包括其他活性氧、小分子生 物硫醇及无机阴离子 $\left(\mathrm{H}_{2} \mathrm{O}_{2}, \cdot \mathrm{OH}, \mathrm{NO}, \mathrm{O}_{2}^{--}, \mathrm{F}^{-}, \mathrm{Cl}^{-}\right.$, $\mathrm{Br}^{-}, \mathrm{I}^{-}, \mathrm{SO}_{4}^{2-}, \mathrm{NO}_{3}^{-}, \mathrm{HCO}_{3}^{-}, \mathrm{S}^{2-}, \mathrm{SCN}^{-}, \mathrm{C}_{2} \mathrm{O}_{4}^{2-}$, $\mathrm{OAc}^{-}, \mathrm{N}_{3}^{-}$, Cys, $\mathrm{Hcy}, \mathrm{GSH}, \mathrm{CN}^{-}, \mathrm{HSO}_{4}^{-}, \mathrm{HS}^{-}$)在内的 共计 22 种干扰物, 结果如图 4 中所示, 从中发现, 当探 针溶液中分别加入 90 equiv. 的其他干扰物质时, 探针的 荧光强度几乎没有变化 (黑色柱形图). 而即使探针溶液 中存在 90 equiv. 的干扰物时, 再加入 9 equiv. 的 $\mathrm{ClO}^{-}$后 探针 NAEC 溶液在 $520 \mathrm{~nm}$ 处的苂光强度同样迅速增强 (红色柱形图). 实验结果表明, 探针 NAEC 几乎不与 $\mathrm{ClO}^{-}$以外的干扰物质反应, 其他干扰物的存在并不影 响 $\mathrm{ClO}^{-}$的检测效能, $\mathrm{NAEC}$ 能够实现 $\mathrm{ClO}^{-}$的特异性检 测.

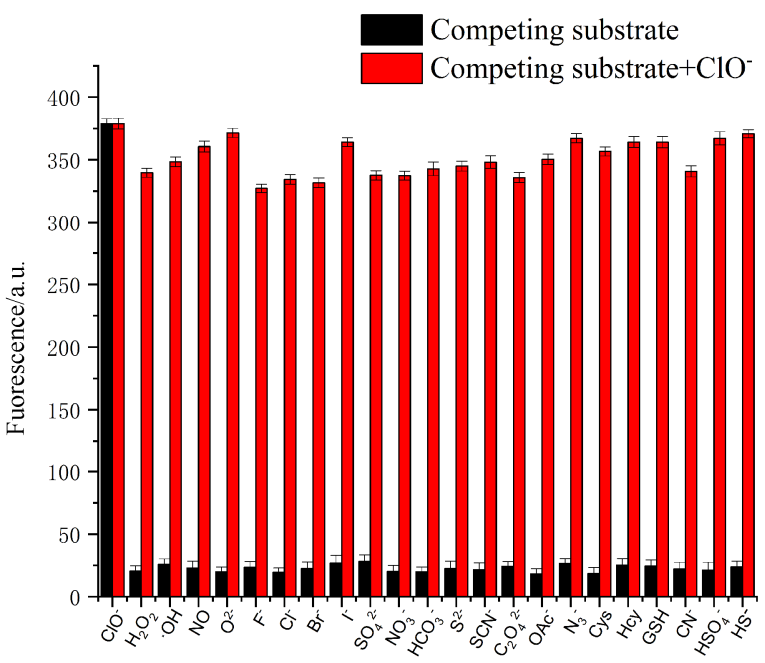

图 4 向存在不同干扰离子 $(90$ equiv.)的探针 NAEC $(5.0 \times$ $10^{-6} \mathrm{~mol} / \mathrm{L}$ ) 溶液中加入 $\mathrm{ClO}^{-}$(9 equiv.) 前后的荧光变化 [DMF/PBS $(V: V=1: 19), \mathrm{pH}=7.4]$

Figure 4 Variation in the fluorescence of NAEC $\left(5.0 \times 10^{-6}\right.$ $\mathrm{mol} / \mathrm{L}$ ) before and after adding 9 equiv. $\mathrm{ClO}^{-}$in the presence of 90 equiv. of various interferents [DMF/PBS $(V: V=1: 19)$, $\mathrm{pH}=7.4]$

\section{5 探针分子的检测机理研究}

为探究探针 NAEC 检测 $\mathrm{ClO}^{-}$的机理, 通过高分辨 质谱对加入 $\mathrm{ClO}^{-}$后的探针进行了测试，结果发现，当探 针分子与 $\mathrm{ClO}^{-}$作用后, 在 $m / z=298.10$ 处显示出明显特 征峰，与化合物 $\mathbf{4}$ 的分子量 $\left(\left[\mathrm{M}+\mathrm{H}^{+}\right] 298.10\right)$ 一致. 同时 在 $420 \mathrm{~nm}$ 激发波长下，化合物 4 在 $520 \mathrm{~nm}$ 处同样有明 显的苂光发射峰(图 5A), 与探针 NAEC 反应后的苂光光 谱相吻合，说明探针与次氯酸作用后产生了化合物 4. 我们综合上述结果提出了可能的作用机理(图 5B), 即探 针分子 $\mathrm{NAEC}$ 中的 $\mathrm{C}=\mathrm{C}$ 双键被次氯酸氧化，生成醛基, 探针 NAEC 原有的 ICT 效应被破坏，产生苂光信号.

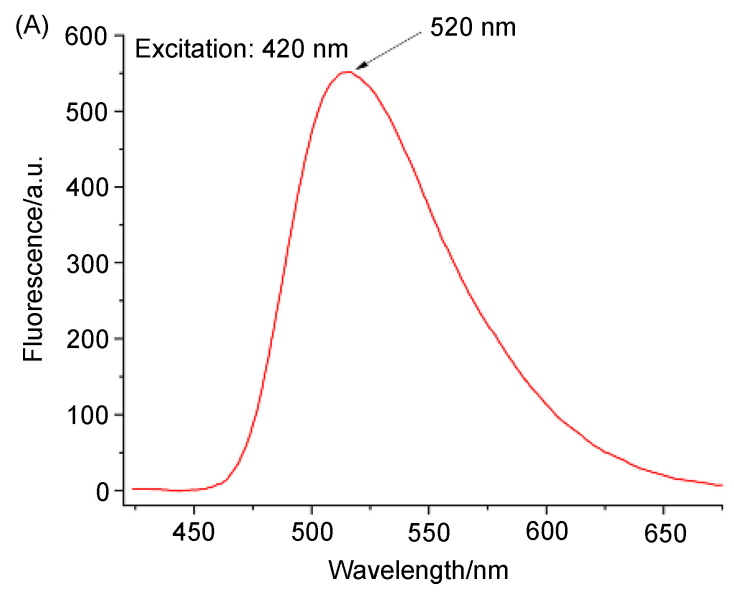

(B)

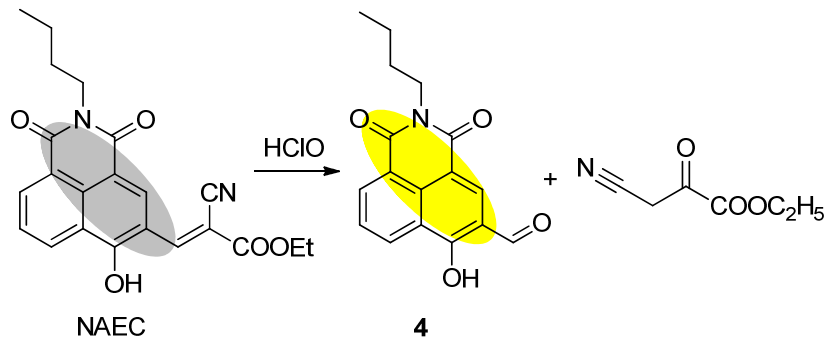

图 5 (A)分子 4 和探针 NAEC 反应后的苂光光谱; (B)探针 $\mathrm{NAEC}$ 对 $\mathrm{ClO}^{-}$的检测机理示意图

Figure 5 (A) Fluorescence spectra of molecule 4 and NAEC and (B) proposed reaction mechanism of probe NAEC towards $\mathrm{ClO}^{-}$

\section{6 细胞毒性实验}

图 6 为脐静脉内皮细胞(HUVEC)与 NAEC 溶液( 0 ～ $30 \mu \mathrm{mol} / \mathrm{L})$ 捊育 $24 \mathrm{~h}$ 后的实验结果，结果表明被测细胞 形态正常，存活率维持在 $90 \%$ 以上，表明探针 NAEC 具 有良好的生物相容性和较低的细胞毒性.

\section{7 探针 NAEC 的体外细胞成像}

为了研究探针 NAEC 在细胞成像中的实际应用效 果, 设置了三组对照实验. 结果表明, 将 HUVEC 细胞 与探针 NAEC 共培养后, 细胞形态良好, 胞内及背景无 


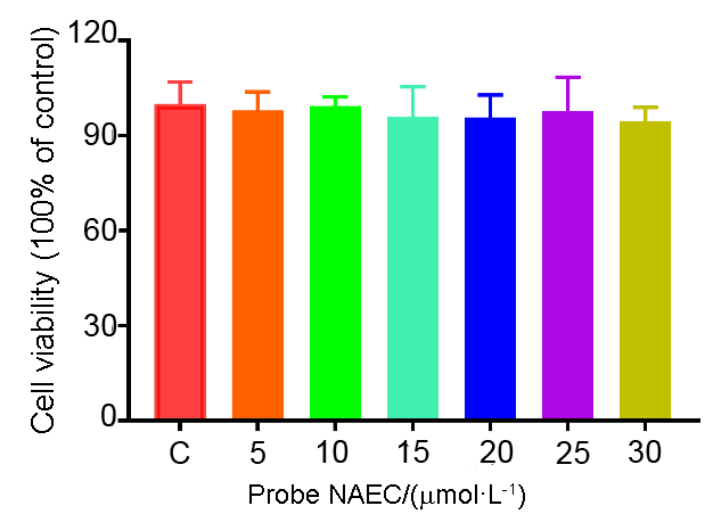

图 6 四甲基偶氮唑盐(MTT)法测得 HUVEC 细胞与探针 NAEC 共孵育 $24 \mathrm{~h}$ 后的细胞存活率(对照组为 $100 \%, \mathrm{C}$ 为对照) Figure 6 Viability of HUVEC cell after incubated with probes NAEC by methyl thiazolyl tetrazolium (MTT) method (C represents control sample, , and the viability is $100 \%)$
荧光信号(图 7A); 在 HUVEC 细胞与探针 NAEC 的培养 体系中加入佛波醇 12-十四酸酯 13-乙酸酯(PMA)后, 细 胞在 PMA 刺激作用下产生次氯酸根, 在苂光显微镜下 可观察到，在细胞内部出现明显的绿色荧光信号(图 7D); 在 HUVEC 细胞与探针 NAEC 的培养体系中加入 次氯酸后，在苂光显微镜下能观察到与 $\mathrm{B}$ 组相同的胞内 绿色荧光(图 7G). 上述结果表明, 探针 NAEC 具有良好 的胞透性，能有效实现细胞内源性 $\mathrm{ClO}^{-}$的苂光显影，具 备实时监测生物体内 $\mathrm{ClO}^{-}$的能力.

\section{2 结论}

设计合成了一种基于菜酰亚胺的新型苂光探针 NAEC，该探针不仅有响应速度快 $(<10 \mathrm{~s})$ 、灵敏度高(检 测限低至 $2.4 \mathrm{nmol} / \mathrm{L})$ 、斯托克斯位移大 $(100 \mathrm{~nm}) 、$ 抗干
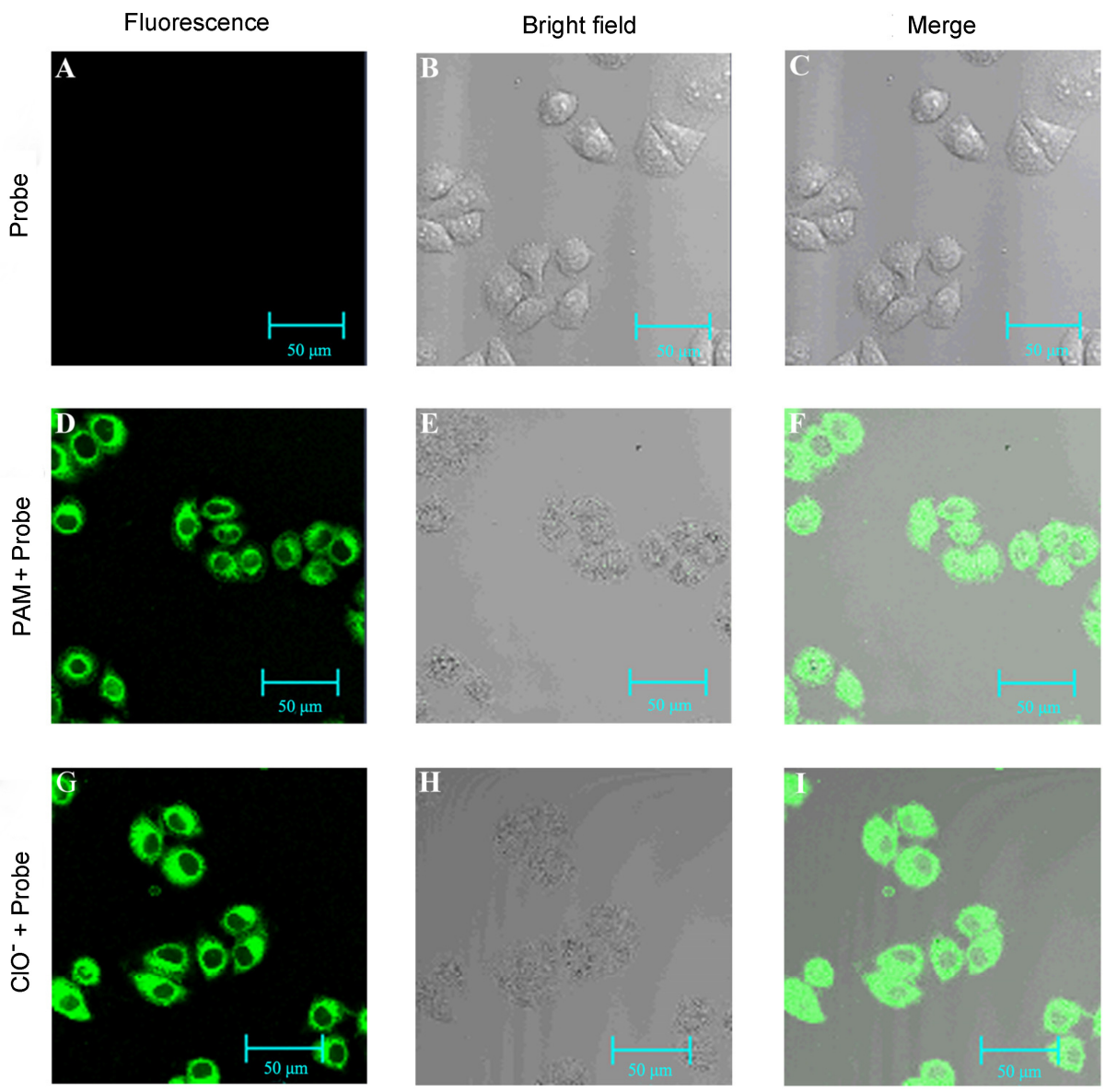

图 7 HUVEC 细胞与探针 NAEC $\left(5.0 \times 10^{-6} \mathrm{~mol} / \mathrm{L}\right)$ 共孵育 $30 \mathrm{~min}(\mathrm{~A}, \mathrm{~B}, \mathrm{C}), \mathrm{HUVEC}$ 细胞经 PMA $(1 \mathrm{mg} / \mathrm{mL})$ 共孵育 $2 \mathrm{~h}$ 后加入探 针 NAEC $\left(5.0 \times 10^{-6} \mathrm{~mol} / \mathrm{L}\right)$ 后继续卯育育 $30 \mathrm{~min}$ 的荧光共聚焦显微图像 $(\mathrm{D}, \mathrm{E}, \mathrm{F}) ; \mathrm{HUVEC}$ 细胞与探针 $\mathrm{NAEC}\left(5.0 \times 10^{-6} \mathrm{~mol} / \mathrm{L}\right)$ 共孵 育 $30 \mathrm{~min}$, 继续加入 9.0 equiv. $\mathrm{ClO}^{-}$共孵育 $30 \mathrm{~min}$ 后的苂光共聚焦显微镜图像 $(\mathrm{G}, \mathrm{H}, \mathrm{I})$ (左：苂光图像; 中：明场图像; 右：重叠图 像)

Figure 7 Optical confocus microscopic images of HUVEC cells incubated 30 min with the NAEC probe $\left(5.0 \times 10^{-6} \mathrm{~mol} / \mathrm{L}\right)(\mathrm{A}, \mathrm{B}, \mathrm{C})$; pretreated for $2 \mathrm{~h}$ with PMA $(1 \mathrm{mg} / \mathrm{mL})$ and incubated with the NAEC probe $\left(5.0 \times 10^{-6} \mathrm{~mol} / \mathrm{L}\right)$ for $30 \mathrm{~min}(\mathrm{D}, \mathrm{E}, \mathrm{F})$ and incubated 30 min with the NAEC probe $\left(5.0 \times 10^{-6} \mathrm{~mol} / \mathrm{L}\right)$, followed by treatment with 9.0 equiv. $\mathrm{ClO}^{-}$for another $30 \mathrm{~min}(\mathrm{G}, \mathrm{H}, \mathrm{I})(\mathrm{left}$ : fluorescence images, middle: bright-field images, right: merge images) 
扰能力强等优点, 而且生物相容性和细胞膜透性较好, 可实现细胞内源性 $\mathrm{ClO}^{-}$的荧光成像, 有望在环境监测 和生物成像领域得到广泛应用。

\section{3 实验部分}

\section{1 仪器与试剂}

Bruker AV-400MHz 型核磁共振波谱仪(瑞士 Bruker 公司); Bruker Agilent 1290-micr OTOF QII 型质谱仪(美 国 Bruker 公司); Hitachi F-4500 型荧光光谱仪(日本日立 公司); Hitachi U-3010 型紫外-可见吸收光谱仪(日本日 立公司); LSM710-型激光共聚焦显微镜(德国蔡司光学); BDSZW-PH 型倒置显微镜(中国重庆奥特公司).

正丁胺、4-澳-1,8-䒬二甲酸酐、氧基乙酸乙酯及乙 醇钠均为分析纯, 购于安耐吉试剂公司. 其余所使用的 试剂和溶剂均为分析纯, 若无特殊说明均为直接使用. 脐静脉内皮细胞(HUVEC)由吉林大学生命科学公共技 术中心提供. 实验用健康新西兰兔由吉林大学基础医学 院细胞生物学系提供. 实验用水为自制超纯水(18.4 $\mathrm{M} \Omega \cdot \mathrm{cm})$.

\section{2 探针 NAEC 的合成}

中间体 1 4 均参照文献[17]合成. 将化合物 4 (2.97 $\mathrm{g}, 10 \mathrm{mmol}$ )与氰基乙酸乙酯 $(1.13 \mathrm{~g}, 10 \mathrm{mmol})$ 共同溶解 于 $50 \mathrm{~mL}$ 无水乙醇中, 回流 $4 \mathrm{~h}$ 后冷却至室温, 减压除 去溶剂, 粗产物经柱层析分离[流动相为 $V$ (二氯甲烷) : $V$ (甲醇) $=20: 1]$, 得 $3.25 \mathrm{~g}$ 紫红色固体, 即为探针分子 NAEC, 产率为 $83 \%$. m.p. $239 \sim 240{ }^{\circ} \mathrm{C} ;{ }^{1} \mathrm{H}$ NMR $(400$ $\left.\mathrm{MHz}, \mathrm{CDCl}_{3}\right) \delta$ : $9.64(\mathrm{~s}, 1 \mathrm{H}), 8.70 \sim 8.63(\mathrm{~m}, 2 \mathrm{H}), 8.57(\mathrm{~s}$, $1 \mathrm{H}), 8.52(\mathrm{~s}, 1 \mathrm{H}), 7.93 \sim 7.79(\mathrm{~m}, 1 \mathrm{H}), 4.29$ (q, $J=7.1 \mathrm{~Hz}$, $2 \mathrm{H}), 4.18 \sim 4.10(\mathrm{~m}, 2 \mathrm{H}), 1.69$ (s, $2 \mathrm{H}), 1.43$ (dd, $J=15.2$, $7.5 \mathrm{~Hz}, 2 \mathrm{H}), 1.37(\mathrm{t}, J=7.1 \mathrm{~Hz}, 3 \mathrm{H}), 0.98(\mathrm{t}, J=7.3 \mathrm{~Hz}$, $3 \mathrm{H}) ;{ }^{13} \mathrm{C}$ NMR (100 MHz, DMSO- $\left.d_{6}\right) \delta: 167.49,163.06$, $162.27,162.17,156.94,154.67,146.44,133.82,130.32$, $129.81,128.42,128.19,122.80,120.68,120.51,119.77$, $117.93,113.90,77.16,76.84,76.53,62.86,61.13,40.42$, 29.93, 24.58, 20.16, 14.13, 13.63; ESI-MS $(\mathrm{m} / \mathrm{z}):[\mathrm{M}+\mathrm{H}]^{+}$ calcd for $\left[\mathrm{C}_{22} \mathrm{H}_{20} \mathrm{~N}_{2} \mathrm{O}_{5}\right]^{+}$393.1445, found 393.1445.

\section{3 溶液的配制}

以 $N, N$-二甲基甲酰胺 (DMF) 为溶剂制备荧光探针 NAEC 原液 $\left(5.0 \times 10^{-4} \mathrm{~mol} / \mathrm{L}\right)$, 以 $\mathrm{pH}$ 为 7.4 的 $\mathrm{PBS}$ 缓冲 溶液为溶剂分别配制浓度为 $1.0 \times 10^{-3} \mathrm{~mol} / \mathrm{L}$ 的 $\mathrm{HClO}$ 溶 液及浓度为 $2.0 \times 10^{-2} \mathrm{~mol} / \mathrm{L}$ 干扰离子溶液 $\left(\mathrm{F}^{-}, \mathrm{Cl}^{-}, \mathrm{Br}^{-}\right.$, $\mathrm{I}^{-}, \mathrm{SO}_{4}^{2-}, \mathrm{NO}_{3}^{-}, \mathrm{S}^{2-}, \mathrm{SCN}^{-}, \mathrm{CO}_{3}^{2-}, \mathrm{NO}_{2}^{-}, \mathrm{C}_{2} \mathrm{O}_{4}^{2-}$, $\mathrm{OAc}^{-}, \mathrm{N}_{3}^{-}$, Cys, Hcy, GSH, CN ${ }^{-}, \mathrm{H}_{2} \mathrm{O}_{2}, \cdot \mathrm{OH}, \mathrm{O}_{2}^{--}$, $\mathrm{NO}$ ). 测试过程中, 分别用上述配置好的 $\mathrm{HClO}$ 和干扰
离子溶液将 $\mathrm{NEAC}$ 原液稀释至 $5.0 \times 10^{-6} \mathrm{~mol} / \mathrm{L}$, 经放置 2 min 后再进行测试.

过氧化氢 $\left(\mathrm{H}_{2} \mathrm{O}_{2}\right)$ 溶液: 取市售质量分数约为 $30 \%$ 的 $\mathrm{H}_{2} \mathrm{O}_{2}$ 水溶液稀释, 采用间接碘量法标定.

羟基自由基 $(\cdot \mathrm{OH})$ 溶液 ${ }^{[18]}$ : 向 $100 \mathrm{mmol} / \mathrm{L}$ 的氯化亚 铁 $\left(\mathrm{FeCl}_{2}\right)$ 溶液中加入 $\mathrm{H}_{2} \mathrm{O}_{2}$ 制得 $100 \mathrm{mmol} / \mathrm{L}$ 的 $\cdot \mathrm{OH}$ 水溶 液.

超氧化自由基 $\left(\mathrm{O}_{2} \bullet^{-}\right)$溶液 ${ }^{[19]}$ : 以 $10 \mathrm{~mL}$ 的二甲基亚 砜(DMSO, 内含少量 18-冠-6)溶解 $71.0 \mathrm{mg}$ 超氧化钾 $\left(\mathrm{KO}_{2}\right)$ 制备 $100 \mathrm{mmol} / \mathrm{L}$ 的 $\mathrm{O}_{2}{ }^{-}{ }^{-}$水溶液.

一氧化氮自由基 $(\mathrm{NO})^{[20]}$ : 用 $20 \mathrm{~mL}$ 去离子水溶解 $298 \mathrm{mg}$ 亚硝基铁氰化钠(硝普钠 SNP)制得 $100 \mathrm{mmol} / \mathrm{L}$ 的 NO 水溶液.

\section{4 细胞毒性试验}

取对数生长期的 HUVEC 细胞悬液接种到 96 孔板 中, 每孔 $100 \mu \mathrm{L}$, 细胞浓度为 $1 \times 10^{4} \mathrm{cell} / \mathrm{L}$, 孵育 $24 \mathrm{~h}$ 后, 加入浓度为 $0,5,10,15,20,25,30 \mathrm{mmol} / \mathrm{L}$ 的探针 NAEC 溶液 [溶剂为含体积分数为 $10 \%$ 的胎牛血清(FBS) 和体积分数为 $1 \%$ 抗生素(青霉素和链霉素)高糖达尔伯 克改良伊格尔培养基(DMEM)], 继续孵育 $24 \mathrm{~h}$. 然后用 四甲基偶氮唑盐(MTT)微量酶反应比色法测定波长在 $490 \mathrm{~nm}$ 处吸光度值 (OD 值). 通过 3 次平行实验取平均 值, 计算细胞存活率.

\section{5 体外细胞成像}

实验分三组: A 组, HUVEC 细胞十探针 NAEC; B 组, HUVEC 细胞 + PMA, 刺激细胞过表达 $\mathrm{ClO}^{-}$) +探针 NAEC; $\mathrm{C}$ 组, HUVEC 细 胞 $+\mathrm{ClO}^{-}$(9 equiv.) +探针 NAEC. 具体操作 ${ }^{[21]}$ 如下: 取对数生长期的 HUVEC 细 胞接种到培养血中, 每孔 $2 \mathrm{~mL}$, 浓度为 $2.0 \times 10^{4}$ cell $/ \mathrm{mL}$, 于 $37{ }^{\circ} \mathrm{C}$, 含有体积分数为 $5 \%$ 的 $\mathrm{CO}_{2}$ 恒温培养 箱中孵育 $24 \mathrm{~h}$. A 组: 以 DMEM 培养基配置 $5 \mu \mathrm{mol} / \mathrm{L}$ 的 探针 NAEC 溶液, 孵育 $30 \mathrm{~min}$. PBS 冲洗后在共聚焦荧 光显微镜下观察细胞荧光成像. B 组: 将 HUVEC 细胞与 $1 \mathrm{mg} / \mathrm{mL}$ 的 PMA 共孵育 $2 \mathrm{~h}$, 之后加入 $10 \mu \mathrm{mol} / \mathrm{L}$ 探针 NAEC 继续孵育 $30 \mathrm{~min}, \mathrm{PBS}$ 冲洗后利用激光共聚焦显 微镜观察荧光成像. $\mathrm{C}$ 组: 在 $\mathrm{A}$ 组细胞体系中追加 $\mathrm{ClO}^{-}$ (9 equiv.), 孵育 $1 \mathrm{~h}$ 后再次用激光共聚焦显微镜观察苂 光成像.

辅助材料(Supporting Information) NAEC 的 ${ }^{1} \mathrm{H}$ NMR, ${ }^{13} \mathrm{C} \mathrm{NMR} 、 \mathrm{HRMS}$ 图谱以及 $\mathrm{NAEC}$ 与 $\mathrm{HClO}$ 反应后的高 分辨质谱这些材料可以免费从本刊网站 (http://siocjournal.cn/)上下载. 


\section{References}

[1] Rutala, W. A; Weber, D. J. Clin. Microbiol. Rev. 1997, 10, 597.

[2] Ye, Z.; Zhang, R.; Song, B.; Dai, Z.; Jin, D.; Goldys, E. M.; Yuan, J. Dalton Trans. 2014, 43, 8414.

[3] Sultana, S.; Foti, A.; Dahl, J. U. Infect. Immun. 2020, 88, e00964-19.

[4] Aiken, M. L.; Painter, R. G.; Zhou, Y.; Wang, G. Free Radicals Biol. Med. 2012, 53, 2308.

[5] Wu, Y.; Wang, J.; Zeng, F.; Huang, S.; Huang, J.; Xie, H.; Yu, C.; Wu, S. ACS Appl. Mater. Interfaces 2016, 8, 1511.

[6] Guo, B.; Nie, H.; Yang, W.; Tian, Y.; Jing, J.; Zhang, X. Sens. Actuators, B 2016, 236, 459.

[7] Yuan, L.; Wang, L.; Agrawalla, B. K.; Park, S. J.; Zhu, H.; Sivaraman, B.; Peng, J.; Xu, Q. H.; Chang, Y. T. J. Am. Chem. Soc. 2015, 137, 5930 .

[8] Shi, D.; Chen, S.; Dong, B.; Zhang, Y.; Sheng, C.; James, T. D.; Guo, Y. Chem. Sci. 2019, 10, 3715.

[9] Xing, P.; Zhang, Z.; Niu, Y.; Qi, Y.; Dong, L.; Wang, C. Chem. Commun. 2018, 54, 9889.

[10] Pak, Y. L.; Park, S. J.; Wu, D.; Cheon, B.; Kim, H. M.; Bouffard, J.; Yoon, J. Angew. Chem., Int. Ed. 2018, 57, 1567.

[11] Liu, S.; Yang, D.; Liu, Y.; Pan, H.; Chen, H.; Qu, X.; Li, H. Sens. Actuators, B 2019, 299, 126937.

[12] Wang, J.; Guo, J.; Dou, L.; Wang, R.; Song, Y.; Yang, Q.; Du, J.; Li,
Y. Chem. Res. Chin. Univ. 2019, 35, 570.

[13] Wang, J. J.; Qi, S. L.; Du, J. S.; Yang, Q. B.; Song, Y.; Li, Y. X. Chem. J. Chin. Univ. 2019, 40, 1397 (in Chinese).

(王金金，戚少龙，杜建时，杨清彪，宋岩，李耀先，高等学校化 学学报, 2019, 40, 1397.)

[14] Cheng, G.; Fan, J.; Sun, W.; Sui, K.; Jin, X.; Wang, J.; Peng, X. Analyst 2013, 138, 6091.

[15] Duan, C.; Won, M.; Verwilst, P.; Xu, J.; Kim, H. S.; Zeng, L.; Kim, J. S. Anal. Chem. 2019, 91, 4172 .

[16] Yao, S. F.; Yao, Y. S.; Zheng, W. B.; Ye, C. Z.; Ying, J.; Lü, G. L.; Li, C. X. Chin. J. Lumin. 2020, 41, 791 (in Chinese). (姚书帆, 尧雨斯, 郑武斌, 叶晨喆, 应杰, 吕光否, 李春霞, 发 光学报, 2020, 41, 791.)

[17] Xiong, H.; He, L.; Zhang, Y.; Wang, J.; Song, X.; Yang, Z. Chin. Chem. Lett. 2019, 30, 1075.

[18] Setsukinai, K.; Urano, Y.; Kakinuma, K.; Majima, H. J.; Nagano, T. J. Biol. Chem. 2003, 278, 3170 .

[19] Gampp, H.; Lippard, S. J. Inorg. Chem. 1983, 22, 357.

[20] Yang, Y.-K.; Cho, H. J.; Lee, J.; Shin, I.; Tae, J. Org. Lett. 2009, 11, 859.

[21] Qi, S. L.; Du, J. S.; Li, R. H.; Li, Q.; Zhu, L. B.; Shi, Y. N.; Wang, X. Y.; Yang, Q. B.; Zhang, G. R.; Li, Y. X. Chin. J. Anal. Chem. 2020, 48, 347 (in Chinese).

(戚少龙，杜建时，李容杭，李强，祝录宝，时亚男，王欣宇，杨 清彪, 张桂荣, 李耀先, 分析化学, 2020, 48, 347.)

(Cheng, F.) 